Ann. Abeille, Ig64, $y$ (I), 63-80.

\title{
POLLINISATION ET FRUCTIFICATION DU CLÉMENTINIER
}

\author{
E. BARBIF:R \\ Station de Recherches sur l'Abeille et les Insectes sociaux, Bures-sur-Ya'etle (Seine-et-Oise) \\ Travatux effectués ì la Station Expérimentale de Boufarik \\ avec la collaboration technique de Mlessieurs WLisit et CoILE.
}

SOMMAIRE

La comparaison détaillée des récoltes fournies par des Clémentiniers ordinaires, dont on connaît la production depuis plusieurs années, et dont la formation des fruits se produit dans des conditions variées, notamment en présence ou en l'absence des abeilles, permet de définir l'importance du rôle de l'abeille dans la fructification de ces plantes.

Ces travaux apportent aussi des précisions nouvelles sur la parthénocarpie chez le clémentinier et l'évolution des fruits formés de diverses façons. Ils fournissent encore un matériel de choix pour aborder un point de botanique encore obscur : l'origine génétique de cet arbre.

\section{IN'TRODUC'TYON}

Dans un important semis de graines de Mandarinier effectué par le frère CLÉNENT en I 898 à l'Orphelinat agricole de Messerghin (Oran), 'TrabuT observa des sujets nettement différents du Mandarinier et dont l'origine hybride lui parut évidente.

Un de ces arbres produisit des fruits d'une telle qualité qu'il fut multiplié dans les vergers sous le nom de Clémentinier de Messerghin en souvenir du frère $C_{\text {L }}$ ÉMENT.

Dès le début, la culture de cet arbre se développa rapidement, d'abord dans la région d'Oran, puis à partir de I926, elle gagna la plaine de la Mitidja, et la plupart des régions agricoles d'Algérie en même temps qu'elle suscitait un grand intérêt au Maroc.

\section{Le Clémentinier et sa fructification.}

'TRabut (I926) désigne comme parents du Clémentinier le Mandarinier et le Bigaradier granito ou Citrus salicifolia, mais d'après la révision de la classification des agrumes par CHAтот (I949) la parenté du Clémentinier proposée par TrABUT deviendrait Citrus reticulata Blanco $\times$ Citrus aurantium $\mathrm{L}$.

Signalons que la preuve de cette parenté n'a encore jamais été apportée malgré l'importance qu'elle présente du point de vue génétique. 
Quoi qu'il en soit, le Clémentinier présentait un tel intérêt arboricole que sa culture s'est répandue très rapidement, mais il est probable que les surfaces qui lui furent attribuées auraient été plus importantes encore si cet arbre n'avait pas déçu les agrumiculteurs par sa fructification qui a pu être qualifiée de "capricieuse $n$.

l)ans ces conditions, de nombreux aboriculteurs abandonnèrent cette variété, mais en même temps, ce qu'il a été convenu d'appeler le "problème du Clémentinier" a provoqué de nombreuses remarques et études, ayant pour but de rechercher les causes de cette improductivité et aussi de proposer cles remèdes.

REBOUR (I950) considérant que l'arbre utilise, rien que pour la floraison, I/4 de ses besoins annuels en azote, préconise de fournir à la plante une fumure azotée sous forme nitrique, un mois avant l'apparition des fleurs.

RENAUD (I954) ajoute que cet apport d'azote doit être accompagné, surtout dans les sols légers, d'une irrigation si la pluviométrie est déficiente.

PETIT (I94I) et BEVANCON (I94I) pratiquent avec succès des incisions annulaires de l'écorce sur les grosses branches et même sur le tronc.

CAPEL caoutchouc.

RENAUd (I954) conseille le greffage du Clémentinier stur Poncirus trifoliata, comme l'avait déjà préconisé 'TRABUT (1926); ce porte-greffe étant moins vigoureux que le Bigaradier, favorise la mise à fruit.

PETIT (I940) signale que le surgreffage d'Orangers, eux-mêmes greffés sur Bigaradier (greffe en sandwich) donne d'excellents résultats.

En fait, les anomalies constatées dans la mise à fruit du Clémentinier semblent être dues à un facteur nutritionnel (déficience du sol en éléments nutritifs ou rapports entre le porte-greffe et le sujet) ; les moyens préconisés pour y remédier et surtout les apports d'engrais azotés se sont montrés efficaces.

Dans un autre ordre d'idée LACARELI,E et MIEDZYRZECKI (I937) constatent que la fleur du Clémentinier est très sensible au froid et au vent ; aussi recommandent-ils de réserver à cet arbre les parcelles bien protégées.

L'aspect climatique du problème de la fructification du Clémentinier ne semble pas négligeable. Les zones maritimes paraissent favorables à cette espèce ; on constate par exemple que le Clémentinier fructifie normalement en Corse ; en Afrique du Nord il est plus fertile dans les régions côtières, ainsi d'ailleurs qu'en Fispagne.

Certains auteurs comme Petrte (I940) Auguste (I954) ont insisté sur le fait que la multiplication du Clémentinier avait été faite sans méthode, et qu'en l'absence de sélection, il se serait créé des clones plus ou moins productifs comme cela s'est produit pour le Citronnier Eureka aux U. S. A. (CuENo'T I947).

C'est ainsi que LARSEN (I942) signale l'existence d'un Clémentinier régulièrement productif et plus précoce que le Clémentinier ordinaire ; cet arbre fut multiplié sous le nom de Clémentinier Monréal. Mais Auguste (I)54), s’il confirme les observations de LARSEN, constate que les fruits de cette variété contiennent 4 fois plus de pépins, ce qui diminue leur valeur.

Énfin, d'autres auteurs ont envisagé le " problème du Clémentinier » sous l'aspect de la pollinisation.

REBOUR (I945) signale la présence de fleurs mal conformées en raison des conditions de milieu; il pense que ces fleurs ont une fertilité réduite. Maurr (I945) cons- 
tate que ces fleurs sont fertiles et COSTE et GAGNARD (I956) obtiennent, par la pollinisation artificielle, un taux de nouaison plus élevé qu'avec les fleurs normales.

Par ailleurs, la plupart des auteurs n'ont pas manqué de signaler que les fruits du Clémentinier ont un nombre de pépins très variable; on signale par exemple o à 20 ou 25 pépins pour des fruits récoltés sur la même branche.

Les travaux de Lacarelife et Mredzyrzecki (I937), Péreau Leroy (I95I), Coste et GAGNaRD (I956) ont montré que la vitalité du pollen de Clémentinier ne peut ètre mise en cause. En effet, il est en grande partie normalement constitué et son taux de germination est largement suffisant pour assurer la fécondation.

La semi-stérilité du Clémentinier serait due en définitive, après les causes déjà énumérées, à une auto-incompatibilité plus ou moins prononcée chez les divers clones, mais cette auto-incompatibilité serait en partie compensée par la possibilité que possède le Clémentinier de développer des fruits parthénocarpiques comme le font la plupart des agrumes.

Considérant la pollinisation par les abeilles, I,ACARELLE et MIEDzYrzecki (1937) ont voulu évaluer leur rôle dans la formation des fruits. Ils ont remarqué que d'une façon générale, elles sont favorables à la fructi fication grâce à la pollinisation croisée qu'elles assurent avec le pollen d'autres agrumes, mais que le nombre de pépins est plus élevé qu'en autopollinisation avec le propre pollen du Clémentinier. Ils considèrent encore que, même dans les plantations homogènes, la présence des abeilles est utile mais ils n'apportent pas, à cette question, toute la précision nécessaire.

Coste et GAGNARD (I956) signalent avoir isolé sous cage un Clémentinier Monréal avec des abeilles et avoir obtenu des fruits à pépins abondants (o à 30).

Perrit (I940) ayant procédé au même essai, mais sans abeilles, sur un Clémentinier ordinaire, constate que la fructification est nulle, alors que LACARELLE et MiEdZYRZECKI (I937) avaient obtenu des fruits sans toutefois en préciser le nombre.

Enfin HORN et TODD (I958), reprenant une expérience de ces derniers chercheurs, montrent que les Clémentiniers ont une production accrue si la pollinisation croisée effectuée par les abeilles est faite avec du pollen d'Oranger, mais ils constatent aussi que les pépins sont plus nombreux.

En conclusion, on peut dire que le problème de la fructification du Clémentinier est des plus complexes. Fn effet, cette fructification est sous la dépendance de plusieurs facteurs qui sont tous plus ou moins déterminants. Toutefois, si la plupart des facteurs relevant de la pratique arboricole ont été étudiés, à juste raison, avec beaucoup de soins, il nous a semblé que le facteur pollinisation, et plus particulièrement le rôle de l'abeille, n'avait pas été considéré avec toute l'attention désirable.

C'est pour combler cette lacune et en opérant dans des conditions bien définies que des travaux ont été entrepris en I 962 à la Station expérimentale de Boufarik afin d'étudier quelques problèmes relatifs à la parthénocarpie, à la pollinisation et au développement des fruits chez le Clémentinier.

\section{CONDITIONS DES ESSAIS}

Nous disposions, pour nos essais, d'une parcelle portant I I 6 clémentiniers ordinaires, greffés sur Poncirus trifoliata, plantés en I94I en terre argileuse avec plan d'eau à faible profondeur. Les arbres reçurent de 1951 à 1957 des "greffes-pont " avec bigaradier a fin de leur donner plus de vigueur 
et améliorer les rendements. Depuis cette date d'ailleurs la production n'a cessé de progresser.

La parcelle considérée est en " non culture ", technique favorable à la production des fruits dans les conditions du sol de cette parcelle et chaque arbre reçoit en outre une fumure minérale répondant annuellement à la formule suivante en chacun des éléments purs :

$$
\mathrm{N}: 1,044 \mathrm{~kg}-\mathrm{P}: 0,332 \mathrm{~kg}-\mathrm{K}: 0,720 \mathrm{~kg}
$$

Parmi ces arbres, les relevés des récoltes (1) permirent de découvrir six arbres dont la moyenne de production des sept dernières années s'établissait à des valeurs très voisines (voir $A$ au tabl. 6).

En avril I962, avant l'apparition de toute fleur, 4 arbres furent recouverts de cages grillagées, selon une technique maintenant classique utilisée pour tous les travaux sur la pollinisation. Les cages sont constituées par des cadres en bois de $1,50 \mathrm{~m} \times 3 \mathrm{~m}$ garnis de toile métallique fine et qui peuvent s'assembler dans tous les sens (fig. I).

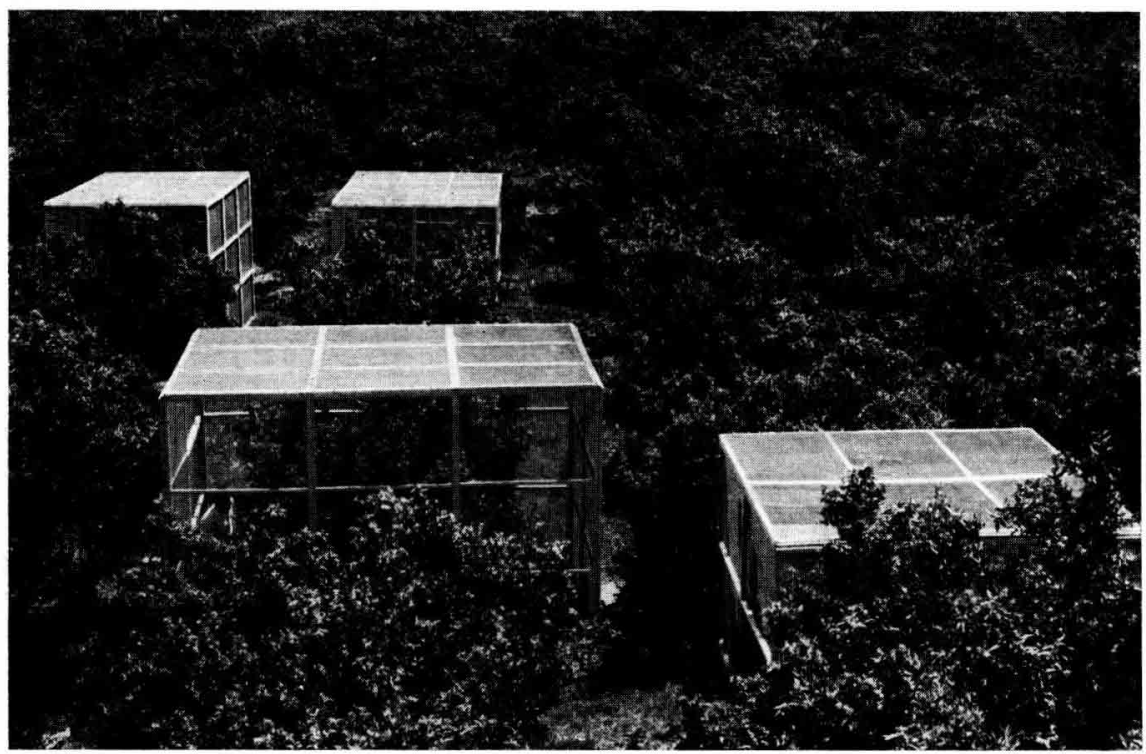

FIG. I. - Vue générale des cages installées dans une parcelle plantée én Clémentiniers

Dans le cas présent, les cages constituées par ro ou I $_{3}$ cadres restèrent en place pendant trute la floraison et ne furent démontées qu'en juin, c'est-à-dire à une époque où toutes les fleurs, méme les plus tardives, avaient complètement disparu.

Les arbres $\mathrm{n}^{\circ} 2666$ et $\mathrm{n}^{\circ} 268 \mathrm{I}$ ayant la même production moyenne furent réservés à l'étude de l'influence de la pollinisation par les abeilles. A cet effet, l'arbre $n^{0} 2666$ fut débarrassé aprés montage de la cage, de tout insecte pouvant provoquer la pollinisation et la cage ne fut jamais ouverte pendant toute la période des essais, alors que dans la cage de l'arbre $n^{0} 268 \mathrm{I}$ et avant l'apparition de la première fleur, il fut introduit une ruchette contenant environ 2000 abeilles de tous âges installies sur 3 cadres Langstroth dont i de couvain et deux contenant un peu de miel et pollen. De temps en temps, cette petite colonic était renforcée par du couvain naissant.

Afin de contrôler la formation des fruits soit par pollinisation artificielle avec du pollen de divers agrumes, soit sous l'influence de produits à action parthénocarpique, deux autres cages furent installées sur les arbres $\mathrm{n}^{\mathrm{0}} 2678$ et $\mathrm{n}^{\mathrm{0}} \mathbf{2 6 9 4}$.

Quant aux arbres $n^{\circ} 2695$ et $n^{\circ} 2745$, ils furent réservés à l'étude de la pollinisation dans les conditions naturelles.

(1) Relevés effectués par M. Bionibel, alors Directeur de la Station, que je remercie pour toutes les informations qu'il a bien voulu me foumir au sujet des agrumes et de leur culture. 


\section{RÉSULTATS}

\section{I) Intensité du butinage sous cage et en plein air}

Les observations ont été faites en procédant à des dénombrements répétés du nombre de fleurs butinées pendant un même laps de temps sur des bouquets de rameaux fleuris présentant un volume et une floraison aussi voisins que possible.

Sous cage, le nombre moyen des visites sur les fleurs est de 6 à 7 fois plus grand qu'en plein air et l'intensité du butinage est à peu près constante au cours de la journée, alors qu'en plein air on note un ralentissement pendant les heures les plus chaudes.

Dans ces conditions, l'intensité du butinage sous cage peut être évaluée approximativement à ro fois ce qu'elle est en plein air ; c'est-à-dire qu'un défaut de pollinisation est exclu.

Ajoutons encore qu'en plein air, nous n'avons pas remarqué la présence d'insectes pollinisateurs autres que les abeilles, à l'exception d'un seul individu hyménoptère mais qui n'a pu être capturé.

Ce manque de pollinisateurs autres que l'abeille est peut-être dû à la nature argileuse des sols de la Mitidja ou à leur humidité, mais c'est un fait qui démontre bien que, dans ce très important centre de production, l'abeille est le seul insecte pouvant assurer la pollinisation du Clémentinier et, sans aucun doute, la pollinisation des agrumes en général.

\section{2) Aspect de la floraison}

Dès le début de la floraison, on constate sous les arbres butinés, en plein air ou sous cage, que les pétales des fleurs tombent très rapidement et qu'ils jonchent le sol en abondance, alors que dans le même temps, les arbres non butinés conservent leurs fleurs plus longtemps et que la chute des pétales ne se produit qu'avec un retard de 2 à 3 jours.

La chute des pièces florales enregistrée sous les arbres butinés est favorisée par l'action mécanique des abeilles sur les pétales et les étamines, lorsqu'elles sont à la recherche du nectar. Toutefois, la pollinisation hâte encore cette chute car elle détermine chez la fleur des réactions physiologiques qui rendent caduques les pièces florales devenues inutiles (VAZART, I955; BARBIER, I962).

\section{3) Pollinisation à la main}

Coste et GAGNARD (I956) ont procédé à des pollinisations à la main sur des fleurs de Clémentinier et citent des taux de nouaison très variables selon les pollens utilisés. Considérant le nombre élevé de variétés appartenant aux diverses espèces en collection à la Station, nous avions envisagé de procéder à des pollinisations très diverses sur l'arbre $n^{\circ} 2694$ protégé par une cage. Malheureusement, les événements ne nous permirent de procéder qu'à 4 séries de ces pollinisations. 
La technique utilisée était la suivante : dans un bouquet fleuri venant d'être cueilli sur un arbre appartenant à une variété étalonnée en collection, une étamine fraîchement ouverte était prélevée avec des pinces et mise en contact avec le stigmate d'une fleur normalement constituée et dont les étamines n'étaient pas encore ouvertes. L'arbre étant sous cage à l'abri des insectes, il ne pouvait y avoir de risque de pollinisation indésirable.

Les résultats obtenus dans ces conditions et portés au tableau I confirment dans leur ensemble ceux obtenus par CosTE et GAGNARD (I956) et démontrent encore que :

$\left.I^{0}\right)$ Les fleurs normales sont fertiles, qu'elles sont facilement fécondables par certains pollens et que la fécondité des pièces femelles de la fleur ne peut être mise en doute.

TABLEAU I

Pollinisation à la main

Clémentinier Messerghin, arbre $n^{0} 2694$ (2 mai 1962, Boufarik)

\begin{tabular}{|c|c|c|c|c|c|}
\hline \multirow{2}{*}{$\begin{array}{l}\text { Espèce, variété et } n^{\circ} \text { de } \\
\text { l'arbre producteur du pollen }\end{array}$} & \multirow{2}{*}{$\begin{array}{c}\text { Nombre de } \\
\text { fleurs pollinisées }\end{array}$} & \multicolumn{2}{|c|}{ Fruits formés } & \multirow{2}{*}{$\begin{array}{c}\text { Nombre } \\
\text { de pépins : } \\
\text { max. et min. }\end{array}$} & \multirow{2}{*}{$\begin{array}{l}\text { Nombre } \\
\text { moyen de } \\
\text { pépins }\end{array}$} \\
\hline & & Nombre & $(\%)$ & & \\
\hline Tangerine DANCY 2112.. & 30 & 26 & 86,6 & 19 à 30 & 26,15 \\
\hline Bigaradier GRANITO 1519. & 12 & 10 & 83,3 & 22 à 29 & 25,30 \\
\hline Citronnier EUREKA 1035. & 18 & 5 & 27,7 & 8 à 19 & 13,60 \\
\hline Témoins & & & & & \\
\hline Fleurs non fécondées.... & 54 & 1 & 1,8 & néant & 0 \\
\hline Clémentinier $2694 \ldots \ldots$ & 50 & 3 & 6 & 2 à 7 & $t_{ \pm}$ \\
\hline
\end{tabular}

$\left.2^{\circ}\right)$ En l'absence de pollen, il peut se former quelques fruits parthénocarpiques aspermes.

$3^{\circ}$ ) Le pollen de Clémentinier est peu efficace pour la formation du fruit (taux de notuaison bas et nombre réduit de pépins).

En conséquence, la présence d'un agent pollinisateur est très favorable à la fructification du Clémentinier.

\section{4) Formation et grossissement des fruits}

Au début du mois d'août, alors que la "chute de juin "était faite, on pouvait relever sur les arbres sans abeilles la présence d'assez nombreux fruits dont la formation parthénocarpique ne pouvait être confirmée qu'après dépouillement des divers résultats et notamment du dénombrement des pépins. Toutefois, un comptage démontrait que les fruits étaient beaucoup plus nombreux sur les arbres pollinisés que sur les arbres protégés des abeilles et que, de plus, dans le premier cas, les fruits étaient plus gros.

Afin de définir la grosseur de ces fruits et pour suivre leur grossissement au 
cours de la végétation, la technique suivante fut utilisée : sur chaque arbre à étudier, 20 fruits, répartis à la périphérie de la frondaison à raison de cinq par point cardinal, sont répérés et numérotés. De semaine en semaine, leur circonférence est soigneusement déterminée à l'aide d'un ruban gradué (1) et leur diamètre moyen est ensuite calculé. Dans chaque série et sur chaque fruit, les grossissements observés sont extrêmement voisins les uns des autres, aussi une moyenne calculée sur 20 fruits est-elle très suffisante.

Résultats : afin de mieux faire apparaître les différences de développement entre les séries, les mesures portées au tableau 2 ont été calculées par rapport aı diamètre moyen des fruits normalement pollinisés pris comme référence à la date de la mesure.

On y trouvera aussi les diamètres de séries de fruits obtenus par pollinisation à la main.

\section{TABLEAU 2}

Influence du mode d'obtention des fruits sur leur diamètre, leur grossissement et sur le nombre des pépins

\begin{tabular}{|c|c|c|c|c|c|c|c|}
\hline \multirow{2}{*}{ Formation des fruits et $n^{\circ}$ de l'arbre } & \multirow{2}{*}{$\begin{array}{l}\text { Nombre } \\
\text { moyen de } \\
\text { pépins par } \\
\left.\text { fruit ( }{ }^{(}\right)\end{array}$} & \multicolumn{2}{|c|}{$\varnothing$ au 15 août } & \multicolumn{2}{|c|}{$\varnothing$ au 29 août } & \multicolumn{2}{|c|}{$\begin{array}{l}\varnothing \text { au } 22 \text { no- } \\
\text { vembre }\end{array}$} \\
\hline & & $(\mathrm{mm})$ & $(\%)$ & $(\mathrm{mm})$ & $(\%)$ & $(\mathrm{mm})$ & $(\%)$ \\
\hline $\begin{array}{c}\text { Parthénocarpie }\left(\mathrm{n}^{\circ} 2666\right) \ldots \ldots \ldots \ldots \ldots \\
\text { Pollinisation par les abeilles }\end{array}$ & 0,06 & 25,76 & 87,20 & 29,70 & 89,08 & 50,93 & 97,92 \\
\hline $\begin{array}{l}\text { Autopollinisation }\left(\mathrm{n}^{\circ} 2681\right) \ldots \ldots \ldots \ldots \ldots \\
\text { En plein air (n' } 2695) \ldots \ldots \ldots \ldots \ldots \\
\text { Pollinisation à la main (arbre 2694) } \\
\text { avec pollen de }\end{array}$ & $\begin{array}{l}1,21 \\
5,21\end{array}$ & $\begin{array}{l}28,69 \\
29,54\end{array}$ & $\begin{array}{l}97,12 \\
100\end{array}$ & $\begin{array}{l}32,42 \\
33,34\end{array}$ & $\begin{array}{l}97,25 \\
100\end{array}$ & $\begin{array}{l}51,28 \\
52,01\end{array}$ & $\begin{array}{l}98,59 \\
100\end{array}$ \\
\hline $\begin{array}{l}\text { Citronnier EUREKa } \ldots \ldots \ldots \ldots \ldots \ldots \ldots \ldots \\
\text { Bigaradier Granito } \ldots \ldots \ldots \ldots \ldots \ldots \ldots \ldots \\
\text { Tangerine Dancy } \ldots \ldots \ldots \ldots \ldots \ldots\end{array}$ & $\begin{array}{l}13,60 \\
25,30 \\
26,15\end{array}$ & & & $\begin{array}{l}42,20 \\
40,68 \\
42,14\end{array}$ & $\begin{array}{l}126,57 \\
122,01 \\
126,39\end{array}$ & $\begin{array}{l}64,87 \\
65,06 \\
67,67\end{array}$ & $\begin{array}{l}124,72 \\
125,09 \\
130,10\end{array}$ \\
\hline
\end{tabular}

(1) Le nombre moyen de pépins est calculé sur la totalité des fruits récoltés sur l'arbre ou sur les fruits de la série considérée.

Dans ce tableau, on peut remarquer que :

a) Dès le I5 aoùt, il existe une grande différence de grossissement entre les fruits des 3 arbres. Les fruits formés en autopollinisation ( $\mathrm{n}^{\circ}$ 268I) ont un diamètre légèrement inférieur à celui des fruits de référence (no 2695) mais les fruits parthénocarpiques ( $n^{0} 2666$ ) sont très nettement plus petits.

b) Le 29 aô̂t, le grossissement des fruits parthénocarpiques apparaît comme plus rapide, mais les fruits pollinisés à la main sont nettement plus gros.

(1) Méthode beaucoup plus précise et plus rapide que celle utilisant un compas de mesure ou un pied à coulisse. De plus, l'emploi du ruban ne risque pas de blesser le fruit. 
Ces observations pourraient faire penser que le pollen, par son abondance ou sa nature, peut avoir une incidence sur le développement du fruit ; mais la cause réelle est la formation des graines comme nous le verrons plus loin.

c) L,e 22 novembre, au moment de la récolte, on peut remarquer que les fruits pollinisés à la main ont continué à se développer sensiblement au même rythme que les fruits de référence (arbre 2695). Par contre, les fruits en autopollinisation par les abeilles (arbre 268I) ont grossi plus rapidement et ont presque rattrapé le diamètre de référence. Quant aux fruits de l'arbre 2666, ils ont eu un développement plus rapide encore puisque de $87,9 \mathrm{p}$. Ioo du diamètre de référence au I $_{5}$ août, ils sont arrivés à près de $98 \mathrm{p}$. Ioo de ce diamètre le 22 novembre.

Les causes de cette différence de rapidité du grossissement doivent être recherchées dans la différence de charge portée par les arbres envisagés. Il est évident que les fruits seront d'autant mieux alimentés qu'ils seront moins nombreux. De plus, la proportion de petits fruits sera réduite et à cet égard le tableau 3 est suffisamment démonstratif.

TABI,EAU 3

Importance des récoltes en fonction du mode de pollinisation

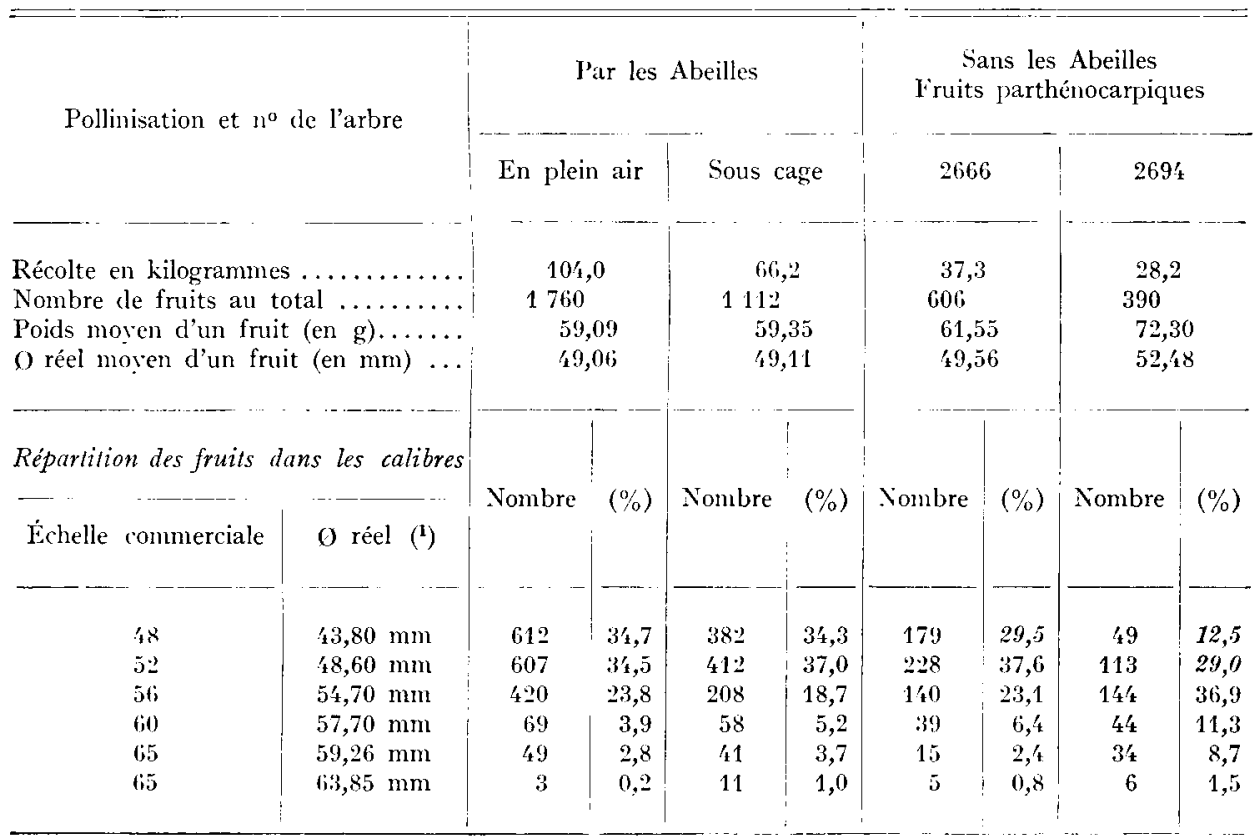

(1) Ie cliamètre réel a été calculé à partir des mesures de circonférence réalisées sur 25 fruits de chaque lot, ajprìs triage par une calibreuse à tapis (type I3lanc).

\section{5) Grosseur des fruits et présence des pépins}

Si les différences de charge sur un arbre peuvent expliquer que les fruits peu nombreux sont mieux alimentés et, de ce fait, grossissent plus vite que ne le font les 
fruits plus nombreux, on constate qu'au moment des premières mesures, c'est-à-dire au I 5 aô̂t, ces fruits d'origine parthénocarpique ont un diamètre plus petit que celui des fruits pollinisés. De plus, malgré un grossissement plus rapide, le diamètre atteint au 22 novembre n'est pas en rapport avec la différence de charge ( $\operatorname{tabl~} n^{0} 3$ ).

Par ailleurs sur l'arbre 2694 protégé des abeilles, les pollinisations à la main ont été faites sur des fleurs très rapprochées les unes des autres et portées sur les jeunes rameaux. La nouaison ayant été excellente, ces fruits se trouvaient dans des conditions très mauvaises pour atteindre de forts diamètres, c'est-à-dire charge locale-

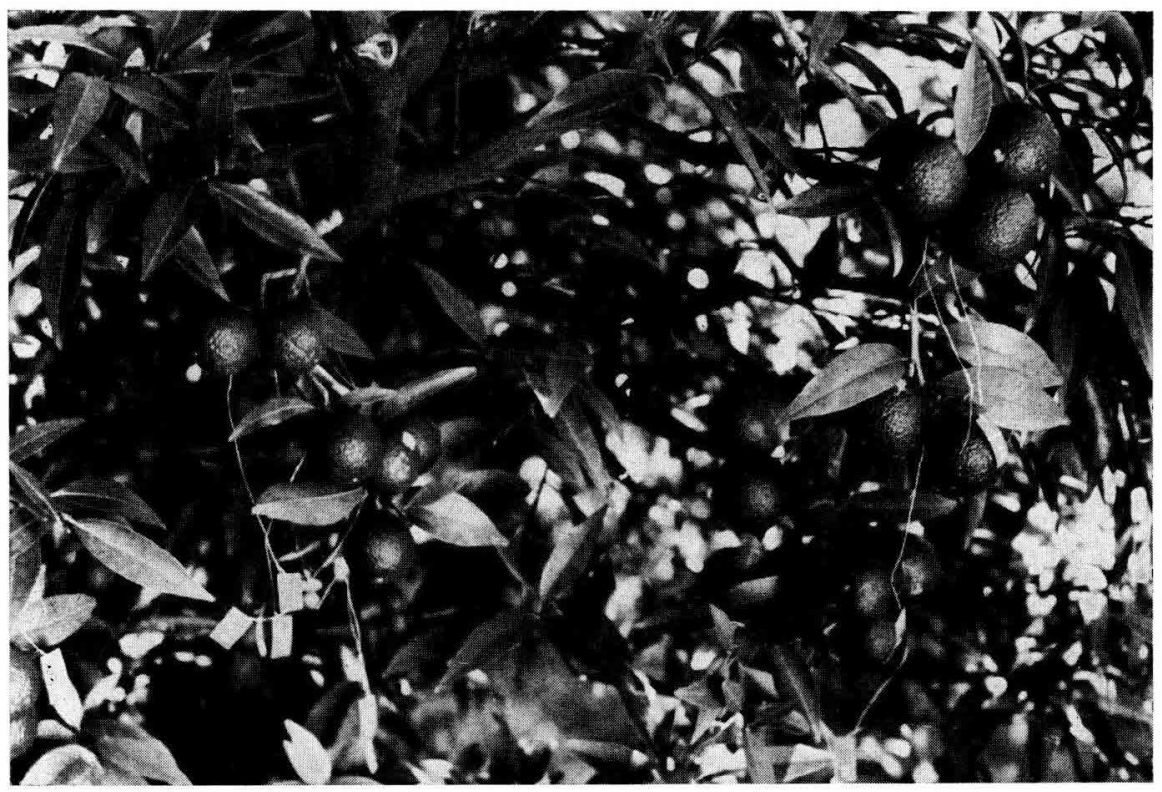

FIts. 2. Clémentinier no 2695. Pollinisation arlificielle avec pollen de Tangerine Dancy Malgré leur nombre sur de petits rameaux, ces fruits ont un diamètre supérieur a celui des /ruits parthénocarpiques $(67,67 \mathrm{~mm}$ contre $52,83 \mathrm{~mm}$

lement très importante et rameaux nourriciers faibles. On remarquera sur la figure 2 de véritables grappes de fruits. Malgré ces conditions défavorables, les diamètres relevés sont très nettement supérieurs au diamètre moyen des fruits parthénocarpiques isolés, formés au voisinage sur le même arbre et se trouvant donc dans des conditions très favorables du point de vue de leur alimentation. La présence des pépins est donc liée à la formation de gros fruits.

Cette constation est également valable lorsqu'on examine en détail la production d'un arbre entier. Pour les 3 arbres étudiés, tous les fruits récoltés ont été épépinés après calibrage et sur la figure 3 le nombre moyen des pépins (normaux ou avortés) contenus dans les seuls fruits contenant au minimum un pépin a été porté en corrélation avec le diamètre réel des fruits (après correction du calibre fourni par la calibreuse). Dans ces conditions : 
a) pour l'arbre $\mathrm{n}^{\circ} 2695$ pollinisé en plein air, plus le diamètre du fruit est grand, plus les pépins sont nombreux ; mais on peut constater que pour les trois premiers calibres le nombre des pépins progresse à un rythme plus lent que pour les gros diamètres.

\section{TABLEAU 4}

Fruits parthénocarpiques et fruits pollinisés à la main sur même arbre

\begin{tabular}{|c|c|c|c|c|c|}
\hline \multirow{2}{*}{ Formation des fruits } & \multirow{2}{*}{$\begin{array}{l}\text { Nombre de } \\
\text { fruits }\end{array}$} & \multicolumn{2}{|c|}{ Nombre de pépins } & \multicolumn{2}{|c|}{ Diamètre (en mun) } \\
\hline & & Écarts & Par fruit & Écarts & Moyen \\
\hline $\begin{array}{c}\text { Parthénocarpie ......... } \\
\text { Pollinisation par }\end{array}$ & 18 & $\begin{array}{c}0-1 \\
\text { (avorté) }\end{array}$ & 0,05 & $49 \quad$ a 57 & $5 \geq, 83$ \\
\hline $\begin{array}{l}\text { Tangerine Dancy ......... } \\
\text { Bigaradier Granito ...... } \\
\text { Citronnier EurEKa ..... }\end{array}$ & $\begin{array}{r}26 \\
10 \\
5\end{array}$ & $\begin{array}{l}22-10 \\
22-29 \\
14-19\end{array}$ & $\begin{array}{l}26,15 \\
25,30 \\
13,60\end{array}$ & $\begin{array}{l}64,6 \text { à } 71,0 \\
62,7 \text { à } 68,4 \\
62,1 \text { à } 70,3\end{array}$ & $\begin{array}{l}67,67 \\
65,06 \\
64,87\end{array}$ \\
\hline
\end{tabular}

b) pour l'arbre no 2666 en autopollinisation stricte (sans abeilles) s'il existe bien quelques rares fruits présentant I ou 2 pépins, toujours avortés d'ailleurs, leur présence accidentelle est sans rapport avec la grosseur du fruit.

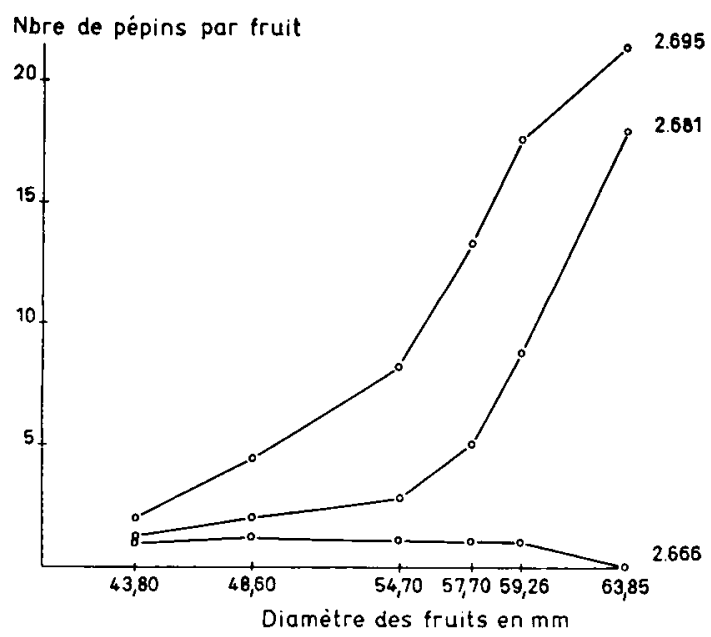

FIG. 3. - Nombre de pépins contemus dans les fruils selon leur grosseur et selon le mode de pollinisation. Arbre $n^{\circ} 2666$ : sous cage en autopollinisation stricte.

Arbre $\mathrm{n}^{\circ} 268 \mathrm{I}$ : sous cage avec abeilles.

Arbre $n^{\circ} 2695$ : en plein air, pollinisation libre avec abeilles.

c) pour l'arbre $268 \mathrm{I}$ en autopollinisation avec les abeilles, l'allure de la courbe est assez voisine de celle de l'arbre $n^{0} 2695$ mais elle est nettement plus basse ; dans sa première partie, au diamètre de 54,70 millimètres correspondent 2,8 pépins contre 8,2 en pollinisation libre, ceci étant dû à la différence de charge portée par les arbres. 
Dans les dernières parties de la courbe, par contre, l'augmentation du nombre de pépins est plus rapide que pour l'arbre $\mathrm{n}^{0} 2695$.

Cette particularité tient sans doute au fait que, lors du dénombrement des pépins, il n'a pas été possible de séparer les pépins avortés des pépins normaux. Des comptages restreints ont montré que les pépins avortés sont plus abondants dans les fruits riches en pépins.

Ces observations sur le diamètre des fruits et sur la présence des pépins apportent une confirmation aux travaux de Niтsch (I954) pour qui les graines diffusent dans les tissus environnants des substances de croissance favorisant le développement $d u$ fruit. Elles expliquent aussi pourquoi LACARELLE et MIEDzyrzecki (I937) avaient déjà noté des discordances apparentes entre le nombre de pépins et le diamètre des fruits selon la provenance de ceux-ci.

\section{TABLEAU 5}

Nombre moyen de pépins (1) par fruit selon les normes utilisées par les services de contrôle à l'exportation

\begin{tabular}{|c|c|c|c|c|c|}
\hline \multirow{2}{*}{$\begin{array}{l}\text { Mode d'obtention } \\
\text { des fruits et } n^{0} \text { de l'arbre }\end{array}$} & \multicolumn{3}{|c|}{ Fruits parthénocarpiques } & \multicolumn{2}{|c|}{ Fruits pollinisés par abeilles } \\
\hline & 2666 & 2678 & 2694 & $\begin{array}{l}\text { Autopolli- } \\
\text { nisation } \\
2681\end{array}$ & $\begin{array}{l}\text { Plein air } \\
2695\end{array}$ \\
\hline $\begin{array}{c}\text { Calibre en } \mathrm{mm} \\
48 \\
52 \\
56 \\
60 \\
65 \\
65\end{array}$ & $\begin{array}{l}0,005 \\
0,09 \\
0,10 \\
0,04 \\
0,1 \\
0\end{array}$ & & & $\begin{array}{r}0,30 \\
0,63 \\
1,67 \\
3,22 \\
6,55 \\
14,54\end{array}$ & $\begin{array}{r}1,92 \\
4,55 \\
8,09 \\
13,30 \\
17,65 \\
21,33\end{array}$ \\
\hline $\begin{array}{l}\text { Nombre moyen sur totalité } \\
\text { de la récolte. }\end{array}$ & 0,06 & 0,01 & 0,09 & 1,21 & 5,21 \\
\hline
\end{tabular}

(1) Pépins normaux et pépins avortés.

D'autre part, si nous considérons les techniques utilisées par les services de contrôle à l'exportation (Répression des Fraudes et O. F. A. L. A. C.) le nombre moyen de pépins contenus dans un fruit de chaque catégorie s'établit selon les données du tableau 5 .

Pour le Clémentinier ordinaire, dit de Messerghin, tout au moins pour les arbres sur lesquels nous avons travaillé, cette étude nous permet encore de confirmer les points suivants :

a) En l'absence de toute pollinisation, il y a production de fruits. Dans ce cas, 
les fruits sont parthénocarpiques; quelques fruits présentent toutefois $\mathbf{I}$ ou plus rarement 2 à 3 pépins mais qui sont tous avortés.

Arbre $\mathrm{n}^{\circ} 2.666$ (sous cage sans abeilles)

\begin{tabular}{|c|c|c|c|c|c|}
\hline Fruits aspermes & $=$ & 576 & ou $95 \%$ & $=$ & 0 pépin \\
\hline Fruits à 1 pépin & $=$ & 25 & $=$ & & 25 pépins \\
\hline Fruits à 2 pépins & $=$ & $4_{4}$ & $=$ & & 8 pépins \\
\hline Fruits à 3 pépins & $=$ & 1 & $=$ & & 3 pépins \\
\hline
\end{tabular}

b) En autopollinisation en présence d'abeilles, l'arbre est loin d'être stérile comme le montre le détail suivant :

Arbre $\mathrm{n}^{\circ} 2$ (is1 (sous cage avec abeille)

\begin{tabular}{|c|c|c|c|c|c|c|}
\hline \multicolumn{7}{|c|}{ Arbre $n^{\circ} 2$ fis (sous cage avec abeille) } \\
\hline Fruits aspermes & $=$ & 689 & $=$ & $61,9 \%$ & $=$ & 0 pépin \\
\hline Fruits avec pépins & $=$ & 423 & $=$ & & & 1362 pépins (1 à 27) \\
\hline au total - fruits & & 1112 & $=$ & & & 1362 pépins \\
\hline
\end{tabular}

Sur ces I 362 pépins, quelques-uns furent coupés au moment de l'épépinage et on constata qu'un certain nombre d'entre eux étaient avortés. La mise en stratification a permis d'obtenir $4 \mathrm{I} 7$ plantules qui furent semées le I5 mai I963 et sur lesquelles nous espérons contrôler la parenté du Clémentinier, laquelle reste bien hypothétique une soixantaine d'années après la découverte de cet arbre étonnant.

En effet, 1'hypothèse de T'RABUT (1926) semble avoir été infirmée par les observations de Webber (cité par CuÉNOT, I947). Il est vrai que dans les deux cas, les graines n'ont pas été obtenues par autopollinisation et les jeunes plants sont des hybrides. La pollinisation de la fleur de Clémentinier par le propre pollen du même arbre pourrait provoquer la ségrégation des caractères des éventuels parents.

c) En pollinisation libre, les fruits sont nettement plus nombreux et les pépins plus abondants, mais il existe tout de même quelques fruits aspermes.

$$
\text { Arbre } \mathrm{n}^{0} 2.695 \text { (en plein air) }
$$

\begin{tabular}{|c|c|c|c|c|c|}
\hline Fruits aspermes & $=$ & 40 & ou $2,3 \%$ & $=$ & 0 pépin \\
\hline Fruits avec pépins & $=$ & 1. 720 & & $=$ & 9.266 pépins $(1$ à 32$)$ \\
\hline au total - fruits & & 1. 760 & & $=$ & 9. 266 pépins \\
\hline
\end{tabular}

La présence de ces fruits aspermes avait déjà été signalée par plusieurs observateurs et nous remarquons ici que leur proportion varie avec le mode de formation des fruits : 2,3 p. Ioo en pollinisation libre en plein air, contre $95 \mathrm{p}$. Ioo en 1'absence d'abeilles.

d) En autopollinisation, bien que la proportion des fruits aspermes soit moins élevée en présence des abeilles qu'en leur absence, nous devrons constater que les abeilles favorisent la formation de ces fruits parthénocarpiques (689 contre 576). Il semble donc que dans la sélection de Clémentinier cultivée à Boufarik, s'il existe une auto-incompatibilité réelle entre le stigmate et le pollen, ce qui se traduit par un nombre, réduit de pépins, la présence du pollen sur le stigmate ou son début de germination ou encore le simple passage des abeilles, suffit à provoquer la formation des fruits parthénocarpiques. 
Quoi qu'il en soit, les résultats obtenus au cours de ces essais montrent que le Clémentinier cultivé à Boufarik possède une autofertilité certaine et ils nous permettent d'envisager avec Petit (I940), Cuḱnot (I947) et Auguste (I954) que l'absence de méthode dans le prélèvement des greffons a pu provoquer la multiplication de clones assez différents en ce qui concerne la productivité, les exigences écologiques, etc. et aussi le degré d'auto-incompatibilité entre le stigmate et le pollen. Pour ce dernier caractère, les deux types extrêmes sont le Clémentinier ordinaire Cadoux et le Clémentinier Monreal signalés par Chapor (1963) dans une toute récente publication.

Nous avions prévu de réaliser les mêmes essais en I963 sur la variété d'orange Washington Navel qui ne produit pas de pollen normalement constitué et dont les fruits sont aspermes. Dans ces conditions, il aurait été possible de définir si le simple butinage des abeilles pouvait, en dehors de toute action possible du pollen, favoriser la formation parthénocarpique des fruits.

Malheureusement, la floraison de la variété Washington Navel a été, cette année, extrêmement faible, aussi nous avons préféré porter nos efforts sur une variété à pollen abondant et fécond, à fruits riches en pépins et dont la floraison a été à peu près normale, l'orange de Blida, afin de contrôler si cette variété peut former des fruits parthénocarpiques en l'absence de toute pollinisation et en autopollinisation avec présence des abeilles.

BLONDEL et BARBIER (I962) avaient constaté qu'en I957 où le mois d'avril avait comporté $I 3$ jours de pluie (I05 millimètres), les fruits contenaient un nombre réduit de pépins, alors qu'en I96r, avec 4 jours de pluie seulement (ro millimètres), les fruits des mêmes arbres en contenaient beaucoup plus.

Il était avancé que cette différence pouvait avoir été provoquée par une diminution de l'activité des abeilles. Les résultats rapportés ci-dessus confirment bien qu'en l'absence des abeilles les pépins sont moins nombreux dans les fruits. Mais l'examen des productions révèle encore que pour les deux années considérées, la fructification calculée sur II6 arbres a été très différente. On note en effet les chiffres suivants qui démontrent d'une façon très nette l'influence de 1'abeille dans la fructification du Clémentier.

\begin{tabular}{|c|c|c|c|c|c|}
\hline \multicolumn{3}{|c|}{ Poids des fruits récoltés : en $\mathrm{kg}$} & \multicolumn{3}{|c|}{ Nombre moyen de pépins par fruit } \\
\hline Année & Au total & Par arbre & Arbres ; 38 & 8 ' & 99 \\
\hline $195 i$ & 7320 & 63,1 & t & 3 & 8 \\
\hline 1961 & 12485 & 107,6 & 12,3 & 15 & 12,7 \\
\hline
\end{tabular}

6) Fructification

Tout comme la présence des pépins dans les fruits, 1'importance des récoltes est en relation avec le mode de pollinisation. Mais avant de procéder à des comparaisons, il est nécessaire d'étudier la production des arbres en expérience, non seu- 
lement en effectuant une moyenne arithmétique des récoltes fournies en 7 ans (I955 à I96I - Voir "A ", tab1.6), mais en examinant année par année leur évolution. Dans ce cas, on constate qu'au cours des 3 dernières années, les productions se sont maintenues à un certain plafond dont les variations n'ont pas dépassé une dizaine de kilogrammes, en plus ou en moins, par rapport aux moyennes portées en " $\mathrm{B}$ " au tableau 6 .

\section{TABLEAU 6}

Influence du mode de formation des fruits sur la production fruitière du Clémentinier ordinaire (1962 Boufarik)

\begin{tabular}{|c|c|c|c|c|c|c|c|}
\hline Présence des abeilles & \multicolumn{3}{|c|}{ Sans abeilles } & \multicolumn{4}{|c|}{ Avec abeilles } \\
\hline Formation des fruits & \multicolumn{3}{|c|}{ Parthénocarpie } & $\begin{array}{l}\text { Autopolli- } \\
\text { nisation }\end{array}$ & \multicolumn{3}{|c|}{ Pollinisation en plein air } \\
\hline No de l'arbre & 2666 & 2678 & $269^{\prime}$ & 2681 & 2695 & 2745 & Parcelle ( $\mathbf{l})$ \\
\hline \multicolumn{8}{|l|}{ Productions moyennes antérieures } \\
\hline$A-1955$ à $1961 \ldots \ldots \ldots \ldots \ldots$ & 84 & 86 & 87 & 84 & 96 & 91 & 一 \\
\hline$B-1959$ à $1961 \ldots \ldots \ldots \ldots \ldots$ & 106 & 110 & 93 & $8 \mathbf{n}^{\prime}$ & 116 & 113 & 117 \\
\hline Résullats 1962 & & & & & & & \\
\hline Production en $1962 \ldots \ldots \ldots \ldots \ldots$ & 37,3 & 36,7 & $28,9 \quad\left(^{2}\right)$ & 66,2 & 104 & 102 & 109 \\
\hline Rapport en $(\%) \frac{1962}{1959 \text { à } 1961} \cdots \cdots$ & 35,2 & $33,4\left(^{2}\right)$ & 33,3 & 78,8 & 89,7 & 90,3 & 93,1 \\
\hline Rapport corrigé . & 39,0 & 37,0 & $3 /, 5$ & 87,5 & & & \\
\hline & & 36,8 & - & & & & \\
\hline
\end{tabular}

(1) Moyenne en ne considérant que les arbres ayant des "greffes-pont ".

(2) Fruits parthénocarpiques seulement.

Ces dernières moyennes sont donc très valables bien qu'elles fournissent des valeurs légèrement différentes d'un arbre à l'autre. En comparant les récoltes de Ig62 avec ces moyennes, on peut, à notre sens, définir valablement l'importance du mode de pollinisation pour la fructification du Clémentinier.

a) En pollinisation libre. Pour la parcelle entière, la moyenne de production des arbres en I962 subit une légère diminution par rapport à la moyenne I959/rg6I, diminution qui est de l'ordre de 7 p. roo. En conséquence, Ig62 n'a pas été une année de production exceptionnellement élevée. Ce point méritait d'être signalé car il permettra de faire plus loin une remarque intéressante.

Les deux arbres témoins $n^{0} 2695$ et $n^{0} 2745$, également en pollinisation libre, accusent aussi une baisse de production. Cette diminution est légèrement plus importante et atteint ro p. Ioo. Nous pensons que cette différence est négligeable et que 
nos chiffres $\left(^{1}\right)$ constituent une base solide pour déterminer le rôle de l'abeille dans la production fruitière du Clémentinier.

Toutes les données chiffrées, concernant la production des arbres, sont indiquées au tableau 6. Après le rapport entre les récoltes de 1962 et celles de la période I959-I96I, un dernier rapport, corrigé en attribuant une production de roo $\mathrm{kg}$ aux deux témoins, nous permet de définir l'importance du rôle de l'abeille dans la fructification.

Cette correction a pour effet de relever légèrement les rapports mais elle permet de chiffrer le rôle de l'abeille avec plus d'exactitude.

b) En autopollinisation avec les abeilles (arbre $\mathrm{n}^{\circ} 268 \mathrm{I}$ ) et malgré un butinage intensif des fleurs, 1'arbre n'a produit que $66,4 \mathrm{~kg}$ soit $87,5 \mathrm{p}$. Ioo de ce qu'il aurait dû produire. La diminution de $\mathrm{I} 2,5 \mathrm{p}$. Ioo n'est peut-être pas très importante, mais elle est déjà sensible.

c) En autopollinisation sans abeilles (arbres no 2666, 2678, 2694). Ia chute de production est considérable puisque ces trois arbres ne produisent en moyenne que $34,9 \mathrm{~kg}$ soit $36,8 \mathrm{p}$. Ioo de ce qu'ils auraient dû produire.

La diminution de production s'établit donc à 63,2 p. Ioo, chiffre qui permet d'évaluer l'importance de l'abeille dans la fructification d'un Clémentinier.

\section{7) Navélisation}

On désigne sous le terme de navélisation une malformation du fruit chez les Citrus et cette malformation est de règle dans les variétés du groupe des Navel. Le fruit navélisé renferme à son sommet et à l'intérieur une deuxième orange plus ou moins développée et même parfois une troisième.

Les abeilles ont été accusées de favoriser la navélisation des fruits. Bien que BOYCE cité par LEROY (I953) signale que cette malformation peut être provoquée par un acarien, nous n'avons jamais constaté, au cours de nos travaux, la moindre navélisation sur les fruits butinés par les abeilles et provenant des arbres en expérience.

Il semble donc que la visite des abeilles soit sans effet sur la navélisation.

\section{8) Coloration des fruits}

Pour définir la couleur qui correspond à la maturité apparente, une échelle de 5 couleurs a été établie :

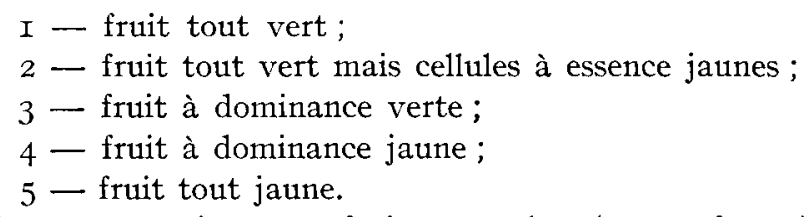

Des relevés ayant porté sur Ioo fruits par arbre $(25$ par face E. S. O. N.) ont permis de constater que chez les fruits pollinisés la couleur est en avance d'une classe.

(1) On voit ici toute l'importance des relevés de production poursuivis régulièrement; ils constituent dans une Station une source inépuisable de renseignements. 


\section{9) Richesse en jus}

Sans vouloir anticiper sur les résultats d'observations effectuées sur la qualité des fruits parthénocarpiques et des fruits pollinisés (1), disons simplement que ces derniers sont nettement moins riches en jus que ne le sont les fruits parthénocarpiques (abstraction faite du poids des pépins).

\section{Io) Relation entre la production fruitière et la floraison de l'année suivante}

Il a été signalé précédemment que la production fruitière de l'année I 962 avait été inférieure de $7 \mathrm{p}$. Ioo environ à la production moyenne des trois dernières années, mais par rapport à la production de l'année I96I la diminution est plus importante encore (Iog contre I29).

Dans ces conditions, r 962 peut être considérée comme une année moyenne qui n'a pas pu fatiguer exagérément les arbres et malgré cela, on constate au moment de la floraison de 1963 que tous les arbres qui étaient en pollinisation normale en plein air ont une floraison extrêmement faible. Le rôle du climat, dont certains facteurs pourraient être responsables de cet état de chose, ne semble pas devoir être retenu car les arbres qui avaient été protégés des abeilles au printemps 1962 et qui de ce fait ont eu une production très réduite, présentent au printemps I 962 une floraison très abondante, comme seuls les Clémentiniers peuvent en porter.

Ajoutons encore que sur l'arbre $\mathrm{n}^{0} 2678$ protégé des abeilles en $\mathbf{1} 962$, et qui a eu une floraison abondante, les branches traitées avec une solution de gibbérelline à 500 p.p.m. $\left({ }^{2}\right)$ et dont la fructification a été abondante en fruits aspermes, ont eu en 1963 une floraison réduite. Il serait donc vain de rechercher uniquement dans les conditions climatiques les causes de la très faible floraison constatée ce printemps I963 sur les Clémentiniers de la Station.

\section{CONCLUSIONS}

De l'ensemble des faits relevés au cours de cette étude, nous pouvons apporter, sur divers points touchant à la fructification du Clémentinier ordinaire dit de Messerghin, les précisions suivantes :

$\left.\mathbf{I}^{0}\right)$ Cet arbre a la propriété de produire des fruits parthénocarpiques en nombre élevé même en l'absence de toute pollinisation; le poids de ces fruits représente 35 à 40 p. 100 de la production d'un arbre pollinisé normalement en plein air, c'est-àdire pollinisé avec des pollens de Clémentinier prélevés sur le même arbre ou sur des arbres voisins, mais aussi très probablement pollinisé avec des pollens provenant d'autres variétés et surtout d'Orangers.

$\left.2^{\circ}\right)$ Le Clémentinier en autopollinisation en présence d'abeilles a une produc-

(1) Publication à paraître en collaboration avec MI. Wuest G. de la Station de Boufarik.

(2) Ce travail fera l'objet d'une note qui sera présentée par M. Wuest G. 
tion de fruits nettement plus élevée, puisque dans nos expériences, elle passe de 36,8 à 87,5 p. Ioo de la production des témoins.

Dans ce cas, les fruits sont plus riches en pépins mais le nombre de fruits aspermes est plus important qu'en l'absence des abeilles. Ainsi le pollen de Clémentinier, s'il est capable d'exciter la formation de fruits parthénocarpiques, ne peut toutefois assurer qu'une fécondation restreinte des ovules.

Bien que la production n'atteigne que 87,5 p. Ioo de la production en pollinisation libre, nous pensons que les fruits présentent une qualité supérieure et que leur écoulement sur les marchés doit en être facilité. Mais, dans la pratique, la production de tels fruits nécessiterait soit l'existence de grandes surfaces en plantation homogène, soit le surgreffage des Citrus voisins autres que ceux appartenant au clone de Clémentinier cultivé, à l'exception, bien entendu, de variétés d'agrumes sans pollen tels que les Orangers du groupe Navel.

$\left.3^{\circ}\right)$ En pollinisation libre, la production atteint son maximum, mais les fruits sont relativement riches en pépins. Or, c'est dans cette condition que sont produites les clémentines dans la plaine de la Mitidja et, en général, dans toutes les régions de culture.

Les résultats obtenus par cette étude montrent que la production des clémentines, et peut-être aussi d'autres agrumes, doit beaucoup à la pollinisation effectuée par l'abeille. Pour un hectare de Clémentiniers cultivé à la Station de Boufarik, où les arbres sont plantés à $5 \times 5 \mathrm{~m}$; la récolte moyenne, dans les conditions définies plus haut. et en pollinisation libre. s'élève à $46800 \mathrm{~kg}$. Sur cette récolte, l'abeille par la seule visite des fleurs, assure la production de $29600 \mathrm{~kg}$ de fruits. En outre, les quatre ruches considérées comme nécessaires à la pollinisation des arbres ont récolté un miel de haute qualité dont le poids peut être évalué, très approximativement, à $120 \mathrm{~kg}$.

Cet exemple démontre l'importance de l'abeille dans la production agricole et aussi l'intérêt qui doit lui être porté dans une agriculture moderne.

Rę̧u pour publication en novembre 1963.

\section{SUMMARY}

TIE POLLINATION AND FRUITING OF TIIE ClementiNe

Six trees having similar average yields over the past few years were chosen in a Clementine plantation. Before the flowers appeared 4 of them were covered with cage nets. A small hive of bees was placed beneath one of these nets, while 2 other netted trees were employed for artificial pollination and the use of parthenocarpic products. The 2 free trees were used for the study of pollination under natural conditions. Results showed that :

r) the Clementine could produce a large number of parthenocarpic fruit even without pollination, but the weight yield was only $35-4 \circ \mathrm{p}$. 100 of that of a tree normally pollinated in the open air ;

2) the Clementine autopollinated in the presence of bees gave a definitely higher yield of more numerous seeded fruit, but the number of seedless fruit was greater than when the bees were absent;

3) production reached its maximum under free pollination. 


\section{RÉFÉRENCES BIBLIOGRAPHIQUES}

Auguste A., 1954. Étude sur la fructification du Clémentinier en Oranie. 3e Congr. Inter. d'Agrum. Méditerr., 2, $28 \mathrm{I}-292$.

BARBIER E. C., 1962. Sur quelques facteurs de la productivité quantitative et qualitative des essences chez les Lavandes (Thèse). Ann. de l'Abeille, 4, $265^{-379}$.

Blondel L., Barbier E., ig62. Le problème des pépins dans les Clémentines. Fruits el Prim. du Maroc, 2. Bevancon, 1941. Station expérimentale d'Agrumiculture de Boufarik. Comptes rendus de l'expérience fruitière en Algérie. Doc. et Rens. agr. Bull. $n^{0} 75$.

Capellades, 1940. Contribution à l'étude du Clémentinier sous forme humoristique. Fruits et Prim. Afrique du Nord, 108, 210.

Сhapot H., 1949. Les Agrumes. Services de l'Horticulture. Rabat.

Снарот H., 1963. Clémentines avec ou sans pépins. Frnits, 18 (5), 259-26r.

Coste A., Cagnard J. M., 1956. Étude sur la pollinisation chez le Clémentinier. Fruits et Primeurs Atrique du Nord, 280, 2+6-252.

Cú́not G., 1947. La tructification capricieuse du Clémentinier. Les Agrumes au Maroc, Dir. de l'Agr., du Commerce et des Forêts au Maroc : 33-37.

Van Horn C. W. et Todd F. E., I955. Bees, bouquets and better tangerines. Progr. Agr. Ariz., 6, (I), i I.

Lacarelle A. et Miedzrrzecki Ch., 1937. Nouvelle contribution à l'étude du Clémentinier au Maroc. Fruits et Primeurs Afr, N., 71, 24-40.

LARSEN G. H., 1942. Improductivité du Clémentinier. C.R. de l'Expériment. fruit. en Algérie. Doc. et Rens. Bull., 92, $15-18$.

Leroy J. F., 1953. I.a navélisation chez le Bigaradier. Les malformations des fleurs et des fruits chez les agrumes. Aspects scientifiques de ces fruits. Rev. Intern. Bot. Appl. et Agr. Trop., 3r1, (2), 414-422.

Mauri N., 1945. Observations sur les anomalies de la floraison du Clémentinier. Comptes rendus de l'Expérimentation fruitière en Algérie. Bull., 118.

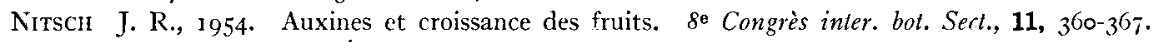

Péreau-Leroy P., 1951. Étude du pollen des Agrumes. 1. F. A. C., Annales 2

Petit R., 1940. Contribution à l'étude du Clémentinier. Fruits el primeurs Afrique du Nord, 102, 47-48.

Petit R., I941. Nouvelle contribution à l'étude du Clémentinier et en particulier de son improductivité. Fruits et primeurs Afr, N., 110, 3-5.

Rebovr II., 194.5. Anomalies de la floraison du Clémentinier. C. R. Exp. Fruits en Algérie, Doc. et Rens, 118.

Rebovr H., 1950. Les agrumes en Afrique du Nord, $3^{\mathbf{e}}$ édition. Union des Syndicats des Producteurs d'Agrumes. Alger.

RENaCD M., 1954. Rapport général sur la chute de juin des Agrumes en relation avec la floraison. 3e Congrès Intern. Agrum. Méditerr., 2, I $23^{-1} 28$.

Trabut, 1926. Ia Clémentine. Les Ifybrides du Cilrus Nobilis. Bull. Inform. Agr., 67.,

Vazard B., 1955. La Parthénocarpie. Bull. Soc. Bot., 7-8, 406-4+3

Le Directeur-Gérant : M. L. CaGNaC

Imprimerie Bussière à Saint-Amand (Cher), France. - 6-5-1964.

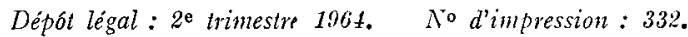

